\title{
Anionic Polymerization of Tertiary Aminostyrenes and Characterization of the Polymers
}

\author{
Kazunori Se, ${ }^{*}$ Masato KiJimA, and Teruo Fujimoto \\ Department of Materials Science and Technology, \\ Technological University of Nagaoka, \\ Kamitomioka 1603-1, Nagaoka, Niigata 940-21, Japan
}

(Received February 10, 1988)

\begin{abstract}
The polymers of three tertiary aminostyrenes: poly $(N, N$-dimethyl-4-vinylphenylamine), abbreviated as PPA; $\operatorname{poly}(N, N$-dimethyl-4-vinylbenzylamine), PBA; and poly( $N, N$-dimethyl-4-vinylphenethylamine), PPTA, were prepared via anionic polymerization in THF at $-78^{\circ} \mathrm{C}$. GPC measurement was carried out to estimate the molecular weights and molecular weight distributions (MWD) of the polymers. A mixture of THF and $N$ methylpyrrolidine $(2 \mathrm{v} / \mathrm{v} \%)$ was used as carrier solvent to avoid the adsorption of the polymers on the surface of the GPC gels. When $n$-butyllithium was used as an initiator, $100 \%$ polymer conversion was attained. The values of $\bar{M}_{w} / \bar{M}_{n}$ were more than 1.20 . The $\bar{M}_{n}$ was equal to $\bar{M}_{k}$, calculated from the amounts of monomers and initiators. No polymer having narrow MWD was obtained, even when $N$-methylpyrrolidine was used as an additive. On the other hand, when cumyl potassium and cumyl cesium were used as initiators, polymers having narrow MWD were produced. The values of $\bar{M}_{w} / \bar{M}_{n}$ were less than 1.08 . The apparent rate constant of polymerization of cumyl cesium for PPA was small, compared with those for PBA and PPTA.

KEY WORDS Tertiary Aminostyrenes / Anionic Polymerization / $\operatorname{Poly}(N, N$-dimethyl-4-vinylphenylamine) / $\operatorname{Poly}(N, N$-dimethyl-4-vinylbenzylamine) / Poly( $N, N$-dimethyl-4-vinylphenethylamine) / Molecular Weight Distribution / Monodisperse Polymers / Adsorption on GPC Gel /
\end{abstract}

Poly(aminostyrenes) are interesting as stable intermediates ${ }^{1}$ of final polymers. For example, poly(amino styrenes) can be used as the backbone chains for graft copolymers, ${ }^{2,3}$ the prepolymers for cross-linked polymers, ${ }^{4}$ and to introduce other functions. ${ }^{1}$

There are two ways to prepare poly(aminostyrenes): (i) polymerization of amino styrenes and (ii) introduction of amino groups to the prepolymers containing no amino groups. For example, $\operatorname{poly}(N, N$-dimethyl-4-vinylbenzylamine) may be prepared by (i) the polymerization of $N, N$ dimethyl-4-vinylbenzylamine and (ii) treatment of chloromethylated poly(styrene) with phthalimide (Gabriel reaction). ${ }^{1}$ The latter way is sometimes convenient to prepare final products without many difficulties. However, amino groups could not be introduced to all the monomer units. Degradation and/or crosslinkage of the polymer chains would proceed during treatment. On the other hand, it is needless to stress the importance of samples having narrow molecular weight distributions in order to study their physical properties. ${ }^{5}$ These facts allow us to study the syntheses of poly(aminostyrenes) via living anionic polymerization.

Amino groups are classified as primary, secondary, tertiary groups and ammonium

\footnotetext{
* To whom correspondence should be addressed.
} 


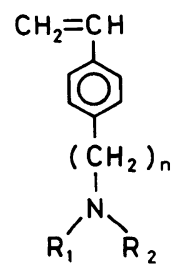

Figure 1. Molecular structure of aminostyrenes. Tertiary amino groups $\left(\mathrm{R}_{1}=\mathrm{R}_{2}=\mathrm{CH}_{3}\right)$ studied in this text are classified into three derivatives: (1) phenyl $(n=0),(2)$ benzyl $(n=1)$, and (3) phenethyl $(n=2)$ amino groups.

salts. The latter can be prepared by quaternization of the amino groups with alkyl halides. ${ }^{6}$ However, amino styrenes having primary and secondary amino groups could not be polymerized via living anionic polymerization due to active hydrogens of the amino groups. It is necessary to protect the active hydrogens of the monomers by an effective protecting group such as an alkyl silyl group. ${ }^{7,8}$

To prepare the stable intermediates of the final polymers, we first synthesized poly(tertiary aminostyrenes): $\operatorname{poly}(N, N$-dimethyl4-vinylphenylamine), $\quad n=0, \quad \mathrm{R}_{1}=\mathrm{R}_{2}=\mathrm{CH}_{3}$, abbreviated as PPA (as shown in Figure 1); $\operatorname{poly}(N, N$-dimethyl-4-vinylbenzylamine), $n=1$, $\mathbf{R}_{1}=\mathbf{R}_{2}=\mathrm{CH}_{3}$, abbreviated as $\mathrm{PBA}^{9,10}$; and $\operatorname{poly}(N, N$-dimethyl-4-vinylphenethylamine), $n=2, \mathrm{R}_{1}=\mathrm{R}_{2}=\mathrm{CH}_{3}$, abbreviated as PPTA. ${ }^{11}$ The purpose of this work was to prepare the poly(aminostyrenes) having narrow molecular weight distributions via living anionic polymerization.

\section{EXPERIMENTAL}

\section{Monomers}

Both monomers of $N, N$-dimethyl-4-vinylbenzylamine and $N, N$-dimethyl-4-vinylphenethylamine were prepared according to previous procedures. ${ }^{9}$

$N, N$-Dimethyl-4-vinylphenylamine was prepared as follows ${ }^{12}$ : methyl iodide $(52 \mathrm{ml})$ in diethylether $(400 \mathrm{ml})$ reacted with magnesium turnings $(20 \mathrm{~g})$ to give a Grignard reagent.
$100 \mathrm{~g}$ of $p-(N, N$-dimethylamino $)$ benzaldehyde dissolved in THF $(200 \mathrm{ml})$ was added to the Grignard reagent. After hydrolysis of the product, $\quad p$-( $N, N$-dimethylamino $)$-1-methylbenzyl alcohol $(106 \mathrm{~g})$ was prepared. The product was throughly dehydrated by heating under vacuum $(2 \mathrm{mmHg})$. Then, $36 \mathrm{~g}$ of monomer were obtained by distillation under reduced pressure: bp was $76-78^{\circ} \mathrm{C} / 2$ Torr and the yield was $55 \%$.

These monomers were fractionally distilled under reduced pressure. They were dried over calcium hydride under reduced pressure, and then purified with octylbenzophenone sodium. Distillation was repeated until the color of the anion remained unchanged during distillation. Finally, they were diluted to about $10 \mathrm{w} / \mathrm{v} \%$ with purified benzene or THF just before polymerization.

\section{Reagents}

An initiator, $s$-butyllithium ( $s$-BuLi), was prepared by a reaction of $s$-butyl bromide with lithium in $n$-heptane. $n$-Butyllithium $(n$-BuLi) was purchased (Nakarai Chemicals, Ltd.) and diluted with purified $n$-heptane. Cumyl potassium (cumyl K) and cumyl cesium (cumyl Cs) were prepared by reactions of cumyl methyl ether with sodium-potassium alloy in THF and cumyl methyl ether with cesium metal in THF, respectively. The concentrations of the initiators were determined by titration with $\mathrm{HCl}$ solution.

Benzene used for the polymerization and $n$ heptane used for the initiator solutions were purified by distillation in vacuo with the addition of $n$-BuLi after the usual purification. THF used for the polymerization and initiator solutions was purified by distillation under reduced pressure in the presence of $\alpha$-methylstyrene tetramer potassium dianions after the usual purification.

$N$-Methylpyrrolidine as an additive to the polymerization solutions was purified with $n$ $\mathrm{BuLi}$ and $\alpha$-methylstyrene tetramer potassium dianions after usual purification. 


\section{Polymerization}

Poly(aminostyrenes) were prepared by living anionic polymerization. The polymerizations were carried out in a sealed glass apparatus under a pressure of $10^{-6}$ Torr. Polymerizations were also carried out at $-78^{\circ} \mathrm{C}$ in THF and at room temperature in benzene, using essentially the same procedures as those in previous works. ${ }^{13,14}$

\section{Molecular Characterization}

All the polymer samples were tested on gel permeation chromatograph (GPC; Model HLC-803, having high resolution columns of G5000H6 $\times 2$ or GMH6 $\times 2$, Toyo Soda Mfg. Co.) to estimate molecular weights and molecular weight distributions. Narrow distribution poly(styrene)s (TSK poly(styrene), Toyo Soda Mfg. Co.) were used as the elution standards. Carrier solvent was THF.

When the polymer samples were held back by adsorption on the surface of the GPC gels due to polar amino groups, $N, N$-dimethylbenzylamine or $N$-methylpyrrolidine as an additive was added to the carrier solvent. The concentration of the additive was in the range of $2-5 \mathrm{v} / \mathrm{v} \%$.

\section{RESULTS}

\section{GPC Measurements}

The polymer samples prepared in this study were tested on a gel permeation chromatograph (GPC) to estimate molecular weights and molecular weight distributions. The GPC chromatogram of PPA showed a sharp peak. However, in Figure 2, the GPC chromatogram of PPTA is abnormal. The fact that the area under the chromatogram is smaller than that expected from the amount of PPTA injected into the GPC indicates adsorption of the polymer onto the surface of the GPC gels. ${ }^{10,15}$ To avoid this, it is necessary to increase the polarity of the carrier solvent to reduce interactions between amino groups of PPTA and the GPC gels. Therefore, $N, N$-dimethylben-

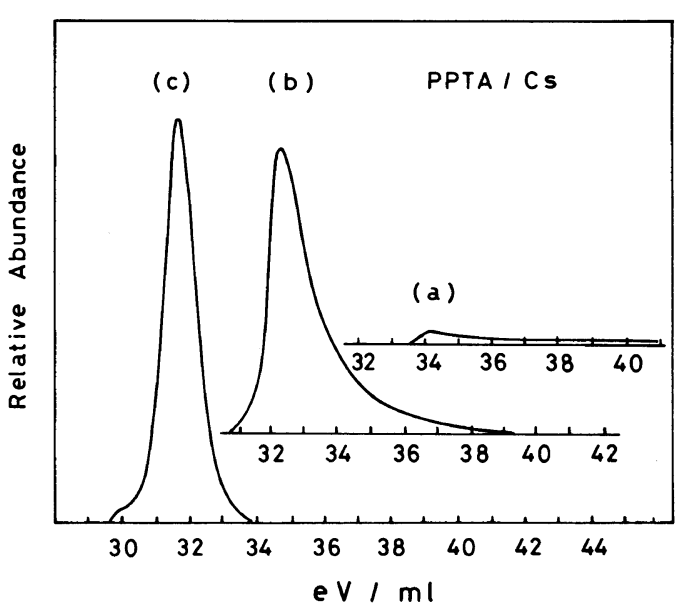

Figure 2. GPC chromatograms of $\operatorname{poly}(N, N$-dimethyl4-vinylphenethylamine), PPTA: $\bar{M}_{n}=7.7 \times 10^{4}$ prepared by using cumyl Cs as an initiator. Carrier solvents of the chromatograms are (a) THF, (b) THF containing $N, N$ dimethylbenzylamine $(2 \mathrm{v} / \mathrm{v} \%)$, and (c) THF containing $N$-methylpyrrolidine ( $2 \mathrm{v} / \mathrm{v} \%$ ), respectively. Flow rate is $1.0 \mathrm{ml} \mathrm{min}{ }^{-1}$, the polymer concentration is $0.05 \mathrm{w} / \mathrm{v} \%$, and RI detector is employed.

zylamine (abbreviated as DBA) was added to THF as the carrier solvent at a concentration of $2 v / v \%$. By use of the carrier solvent, the GPC chromatogram had a single peak, as shown in Figure 2. However, the pronounced tailing of the peak was still observed at the low molecular weight side. The elution volume of the chromatographic peak also shifted to the lower molecular weight side than that predicted from the amounts of the monomer and initiator.

$\mathrm{N}$-Methylpyrrolidine (abbreviated as NMP) has higher basicity than that of DBA. ${ }^{15}$ When a mixture of THF and NMP $(2 \mathrm{v} / \mathrm{v} \%)$ was used as the carrier solvent, the GPC chromatogram showed a sharp peak (Fig. 2). The elution volume of the chromatographic peak was close to the value predicted from the amounts of the monomer and initiator. By use of the carrier solvent having higher NMP content $(5 \mathrm{v} / \mathrm{v} \%)$, the GPC chromatogram was exactly the same as that observed in the mixture of THF and NMP ( $2 \mathrm{v} / \mathrm{v} \%)$. On the other hand, GPC chromatograms of standard poly(styrene)s were 
K. Se, M. Kijima, T. Fujimoto

Table I. Preparation of poly( $N, N$-dimethyl-4-vinylphenylamine), PPA

\begin{tabular}{|c|c|c|c|c|c|c|c|c|c|}
\hline \multirow{2}{*}{$\frac{\text { Monomer }}{\mathrm{moll}^{-1}}$} & \multicolumn{2}{|c|}{ Initiator } & \multicolumn{2}{|l|}{ Solvent } & \multirow{2}{*}{$\frac{\text { Time }}{h}$} & \multirow{2}{*}{$\frac{\text { Conv. }}{\%}$} & \multirow{2}{*}{$10^{-4} \bar{M}_{k}^{\mathrm{a}}$} & \multirow{2}{*}{$10^{-4} \bar{M}_{n}^{\mathrm{b}}$} & \multirow{2}{*}{$\bar{M}_{w} / \bar{M}_{n}^{\mathrm{b}}$} \\
\hline & Name & $10^{4} \mathrm{moll}^{-1}$ & Name & $\mathrm{ml}$ & & & & & \\
\hline 0.22 & $s$-BuLi & 5.16 & Benzene & 220 & 15 & 80 & 5.0 & 2.8 & 1.32 \\
\hline 0.22 & n-BuLi & 4.24 & THF & 210 & 24 & 100 & 7.6 & 8.4 & 1.22 \\
\hline 0.30 & n-BuLi & 7.65 & $\mathrm{THF} / \mathrm{NMP}^{\mathrm{c}}$ & 180 & 24 & 100 & 5.7 & 6.0 & 1.34 \\
\hline 0.22 & Cumyl Cs & 4.40 & THF & 170 & 24 & 40 & 3.0 & 3.6 & 1.09 \\
\hline 0.34 & Cumyl Cs & 6.10 & THF & 120 & 48 & 75 & 6.0 & 6.5 & 1.06 \\
\hline 0.32 & Cumyl Cs & 8.15 & THF & 110 & 48 & 85 & 4.9 & 5.8 & 1.08 \\
\hline 0.35 & Cumyl K & 2.70 & THF & 160 & 24 & 100 & 3.1 & 2.7 & 1.05 \\
\hline 0.31 & Cumyl K & 1.44 & THF & 220 & 24 & 100 & 25.2 & 26.0 & 1.04 \\
\hline
\end{tabular}

a Molecular weights calculated from the amounts of monomer and initiator.

b Molecular weights determined from GPC measurements.

c NMP shows $N$-methylpyrrolidine as an additive. The concentration was $1.4 \mathrm{v} / \mathrm{v} \%$.

not affected by the addition of DBA or NMP to the carrier solvent. A calibration curve determined from standard poly(styrene)s also remained unchanged no matter how the carrier solvent contains DBA $(2 \mathrm{v} / \mathrm{v} \%)$ or NMP (2$5 \mathrm{v} / \mathrm{v} \%$ ). Therefore, the mixture of THF and NMP $(2 \mathrm{v} / \mathrm{v} \%)$ was employed as the carrier solvent in this study.

\section{$\operatorname{Poly}(N, N$-dimethyl-4-vinylphenylamine), PPA}

The results of the Polymerization for PPA are shown in Table I. When $s$-BuLi and benzene were used as the initiator and solvent, respectively, the polymerization solution showed a light red color at room temperature, corresponding to the characteristic color of living carbanions. The solution gradually changed from light red to colorless, and then finally remained colorless after $15 \mathrm{~h}$. The polymer conversion was $80 \%$. The unimodal GPC chromatogram of the obtained polymer was broad and symmetrical about the elution volume. The living carbanions might be deactivated by methyl groups on the amino groups during the chain propagation reaction.

When $n$-BuLi and THF were used as the initiator and solvent at $-78^{\circ} \mathrm{C}$, respectively, the polymerization solution showed a yellow color. The color remained unchanged overnight. When methanol was added to the so-

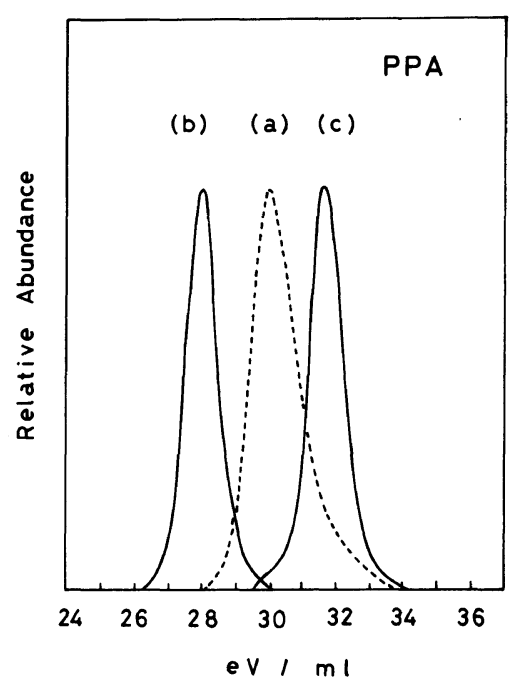

Figure 3. GPC chromatograms of poly $(N, N$-dimethyl4-vinylphenylamine), PPA: (a) $\bar{M}_{n}=8.4 \times 10^{4}$ prepared by using $n$-BuLi, (b) $\bar{M}_{n}=2.6 \times 10^{4}$ by cumyl K, and (c) $\bar{M}_{n}=6.5 \times 10^{4}$ by cumyl Cs as initiators. Carrier solvent is THF. Flow rate is $1.0 \mathrm{ml} \mathrm{min}^{-1}$, the polymer concentration is $0.05 \mathrm{w} / \mathrm{v} \%$, and RI detector is employed.

lution, the solution changed suddenly from yellow to colorless. The polymer conversion was $100 \%$. The molecular weight observed, $\bar{M}_{n}$, was close to the molecular weight expected from the amounts of monomer and initiator, $\bar{M}_{k}$. The GPC chromatogram had a broad peak, as shown in Figure 3. The molecular heterogeniety, $\bar{M}_{w} / \bar{M}_{n}$ value, was $1.22 . N-$ 
Table II. Preparation of poly( $N, N$-dimethyl-4-vinylphenylamine), PBA

\begin{tabular}{|c|c|c|c|c|c|c|c|c|c|}
\hline \multirow{2}{*}{$\frac{\text { Monomer }}{\mathrm{moll}^{-1}}$} & \multicolumn{2}{|c|}{ Initiator } & \multicolumn{2}{|l|}{ Solvent } & \multirow{2}{*}{$\frac{\text { Time }}{h}$} & \multirow{2}{*}{$\frac{\text { Conv. }}{\%}$} & \multirow{2}{*}{$10^{-4} \bar{M}_{k}^{\mathrm{a}}$} & \multirow{2}{*}{$10^{-4} \bar{M}_{n}^{\mathrm{b}}$} & \multirow{2}{*}{$\bar{M}_{w} / \bar{M}_{n}^{\mathrm{b}}$} \\
\hline & Name & $10^{4} \mathrm{moll}^{-1}$ & Name & $\mathrm{ml}$ & & & & & \\
\hline 0.21 & $n$-BuLi & 6.34 & $\mathrm{THF}$ & 120 & 24 & 100 & 5.4 & 4.1 & 1.38 \\
\hline 0.17 & $n-\mathrm{BuLi}$ & 5.64 & $\mathrm{THF} / \mathrm{NMP}^{\mathrm{c}}$ & 150 & 24 & 100 & 5.0 & 3.9 & 1.33 \\
\hline 0.20 & Cumyl K & 9.22 & THF & 200 & 24 & 100 & 3.4 & 3.5 & 1.09 \\
\hline 0.27 & Cumyl Cs & 4.21 & THF & 200 & 3 & 100 & 10.4 & 11.1 & 1.05 \\
\hline
\end{tabular}

a Molecular weights calculated from the amounts of monomer and initiator.

b Molecular weights determined from GPC measurements.

c NMP shows $N$-methylpyrrolidine as an additive. The concentration was $0.9 \mathrm{v} / \mathrm{v} \%$.

Methylpyrrolidine as an additive was also added to the polymerization solution to produce PPA having a narrow molecular weight distribution. ${ }^{16,17}$ The $\bar{M}_{w} / \bar{M}_{n}$ value was still more than 1.30 .

When cumyl Cs and THF were used as the initiator and solvent at $-78^{\circ} \mathrm{C}$, respectively, the polymerization solution remained red overnight. In spite of the low polymer conversion $(40 \%), \bar{M}_{n}$ was close to the $\bar{M}_{k}$. The $\bar{M}_{w} / \bar{M}_{n}$ value was less than 1.10 . In order to complete the chain propagation reaction, the polymerizations were carried out for concentrated solutions of the living ends during $48 \mathrm{~h}$. The polymer conversions were less than $85 \%$. As shown in Figure 3, the GPC chromatogram had a sharp peak. The $\bar{M}_{w} / \bar{M}_{n}$ value decreased from 1.09 to 1.06 with increasing molecular weight.

When cumyl $\mathrm{K}$ and THF were used as the initiator and solvent at $-78^{\circ} \mathrm{C}$, respectively, the polymer conversion was $100 \% \cdot \bar{M}_{n}$ was close to the $\bar{M}_{k}$. As shown in Figure 3 , the GPC chromatogram had a sharp peak. The $M_{w} / \bar{M}_{n}$ value was less than 1.05 . We succeeded in preparing monodisperse PPA samples having $\bar{M}_{n}=2.7 \times 10^{4}$ and $2.6 \times 10^{5}$.

\section{$\operatorname{Poly}(N, N$-dimethyl-4-vinylbenzylamine), $P B A$}

The results of the polymerization for PBA are shown in Table II. When $n$-BuLi was used as the initiator, the polymerization solution remained yellow overnight. The polymer con-

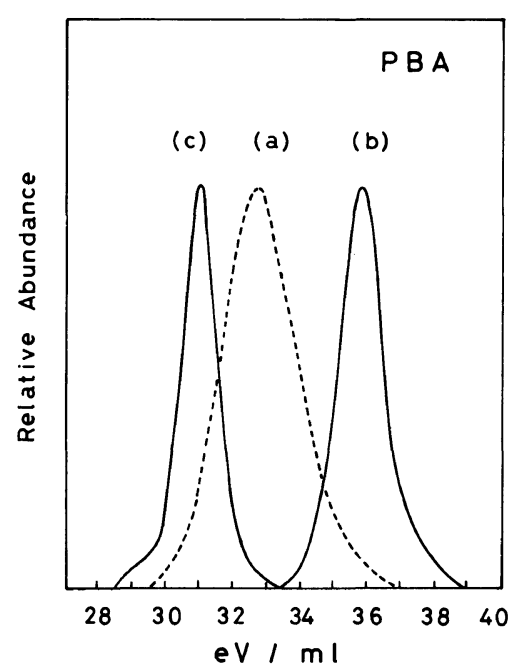

Figure 4. GPC chromatograms of $\operatorname{poly}(N, N$-dimethyl4-vinylbenzylamine), PBA: (a) $\bar{M}_{n}=4.1 \times 10^{4}$ prepared by using $n$-BuLi, (b) $\bar{M}_{n}=3.5 \times 10^{4}$ by cumyl K, and (c) $\bar{M}_{n}=11.1 \times 10^{4}$ by cumyl Cs as initiators. Carrier solvent is THF containing $N$-methylpyrrolidine ( $2 \mathrm{v} / \mathrm{v} \%$ ). Flow rate is $1.0 \mathrm{ml} \mathrm{min}^{-1}$, the polymer concentration is 0.05 $\mathrm{w} / \mathrm{v} \%$, and RI detector is employed.

version was $100 \%$. The $\bar{M}_{n}$ was close to the $\bar{M}_{k}$. As shown in Figure 4, the GPC chromatogram had a broad peak. The $\bar{M}_{w} / \bar{M}_{n}$ value was more than 1.38. $N$-Methylpyrrolidine as an additive did not permit preparation of PBA having a narrow molecular weight distribution.

However, using cumyl $\mathrm{K}$ as an initiator, we prepared a polymer having a narrow molecular weight distribution in $100 \%$ polymer conversion. The $\bar{M}_{n}$ was in good agreement 
Table III. Preparation of poly( $N, N$-dimethyl-4-vinylphenylamine), PPTA

\begin{tabular}{|c|c|c|c|c|c|c|c|c|c|}
\hline \multirow{2}{*}{$\frac{\text { Monomer }}{\text { moll }^{-1}}$} & \multicolumn{2}{|c|}{ Initiator } & \multicolumn{2}{|l|}{ Solvent } & \multirow{2}{*}{$\frac{\text { Time }}{\mathrm{h}}$} & \multirow{2}{*}{$\frac{\text { Conv. }}{\%}$} & \multirow{2}{*}{$10^{-4} \bar{M}_{k}^{\mathrm{a}}$} & \multirow{2}{*}{$10^{-4} \bar{M}_{n}^{\mathrm{b}}$} & \multirow{2}{*}{$\bar{M}_{w} / \bar{M}_{n}^{\mathrm{b}}$} \\
\hline & Name & $10^{4} \mathrm{moll}^{-1}$ & Name & $\mathrm{ml}$ & & & & & \\
\hline 0.16 & $n$-BuLi & 6.22 & THF & 120 & 24 & 100 & 4.5 & 4.1 & 1.22 \\
\hline 0.18 & $n$-BuLi & 6.50 & $\mathrm{THF} / \mathrm{NMP}^{\mathrm{c}}$ & 140 & 24 & 100 & 4.9 & 4.2 & 1.08 \\
\hline 0.17 & Cumyl K & 8.57 & THF & 200 & 24 & 100 & 3.5 & 3.0 & 1.08 \\
\hline 0.21 & Cumyl Cs & 5.14 & THF & 160 & 5 & 100 & 7.1 & 7.7 & 1.04 \\
\hline
\end{tabular}

a Molecular weights calculated from the amounts of monomer and initiator.

b Molecular weights determined from GPC measurements.

c NMP shows $N$-methylpyrrolidine as an additive. The concentration was $1.2 \mathrm{v} / \mathrm{v} \%$.

with the $\bar{M}_{k}$. With cumyl Cs as an initiator, we also prepared PBA having a narrow molecular weight distribution in $100 \%$ polymer conversion. The $\bar{M}_{n}$ was close to the $\bar{M}_{k}$. From the $\bar{M}_{w} / \bar{M}_{n}$ values, the latter appears suitable for preparation of PBA having a narrow molecular weight distribution.

\section{$\operatorname{Poly}(N, N$-dimethyl-4-vinylphenethylamine $)$, PPTA}

The results of the polymerization for PPTA are shown in Table III. When $n$-BuLi was used as the initiator, the polymer solution remained yellow overnight. The polymer conversion was $100 \%$. The $\bar{M}_{n}$ was close to the $\bar{M}_{k}$. As shown in Figure 5, the GPC chromatogram had a broad peak. The $\bar{M}_{w} / \bar{M}_{n}$ value was more than 1.22. $N$-Methylpyrrolidine as an additive permitted preparation of PPTA having a narrow molecular weight distribution.

When cumyl $\mathrm{K}$ and cumyl $\mathrm{Cs}$ were used as initiators, the polymer conversions were $100 \%$. The $\bar{M}_{n}$ were close to the $\bar{M}_{k}$. As shown in Figure 5, the GPC chromatograms had sharp peaks. The $\bar{M}_{w} / \bar{M}_{n}$ value of cumyl Cs system was less than that of cumyl $\mathrm{K}$ system. The former system was well suited for the preparation of PPTA having a narrow molecular weight distribution.

\section{DISCUSSION}

It is well established that nearly monodis-

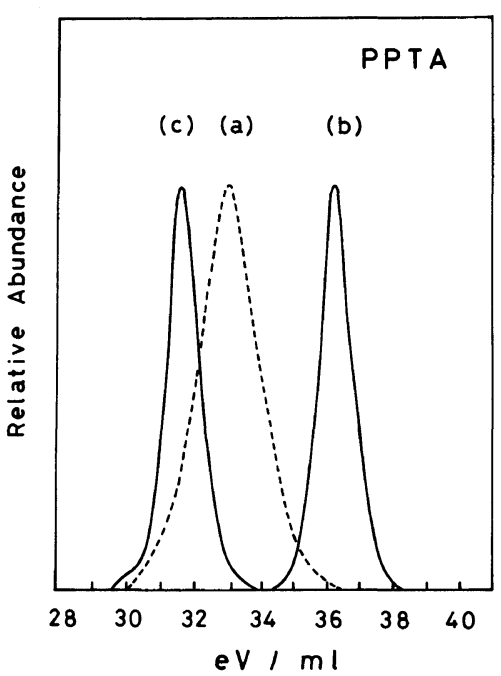

Figure 5. GPC chromatograms of $\operatorname{poly}(N, N$-dimethyl4-vinylphenethylamine), PPTA: (a) $\bar{M}_{n}=4.2 \times 10^{4}$ prepared by using $n-\mathrm{BuLi}$, (b) $\bar{M}_{n}=3.0 \times 10^{4}$ by cumyl K, and (c) $\bar{M}_{n}=7.7 \times 10^{4}$ by cumyl Cs as initiators. Carrier solvent is THF containing $N$-methylpyrrolidine (2 $\mathrm{v} / \mathrm{v} \%$ ). Flow rate is $1.0 \mathrm{ml} \mathrm{min}^{-1}$, the polymer concentration is $0.05 \mathrm{w} / \mathrm{v} \%$, and RI detector is employed.

persed polymers can be obtained by anionic polymerization. To obtain polymers having narrow molecular weight distributions, however, various conditions must be fulfilled in preparing the polymers: (a) "living" polymerization proceeds, (b) the carbanion responsible for anionic polymerization must be stable enough and not react with solvent, side chains of polymers, etc., and (c) the initiation rate constant must be much larger than the propa- 
gation rate constant. We did not specially study the kinetics of the polymerization for tertiary aminostyrenes. Hence, these three conditions could not be confirmed in detail.

However, the facts that the polymerization solutions showed characteristic colors of carbanions and block copolymers of tertiary aminostyrenes and styrene were quantitatively obtained in preliminary preparations ${ }^{4}$ suggest that the "living" polymerization of tertiary aminostyrenes proceeded.

The experimental proofs of condition (b) correspond to the following experimental facts: (1) $100 \%$ polymer conversion is attained and (2) the $\bar{M}_{n}$ is in good agreement with the $\bar{M}_{k}$, being the molecular weight expected from the amounts of monomer and initiator. The experimental proof of condition (c) corresponds to the following experimental fact: (3) the molecular weight distribution is sharp. (However the heterogeneity, $\bar{M}_{w} / \bar{M}_{n}$ value, is dependent on the molecular weight, the $\bar{M}_{w} / \bar{M}_{n}$ value should be less than 1.07.)

Impurities present in the polymerization solutions caused termination, resulting in broad molecular weight distribution and low molecular weight of the polymers. Even when there were no impurities in the polymerization solution, it was difficult to prepare monodisperse polymers having $\bar{M}_{n}$ much more than $10^{6}$, because of the viscous polymerization solutions. Hence, an additional proof of the conditions (b) and (c) corresponds to the following experimental fact: (4) the polymer having a narrow molecular weight distribution can be prepared in a wide range of molecular weight from $10^{4}$ to $10^{6}$.

The $s$-BuLi/benzene system was found unsuitable for the preparation of PPA since experimental fact (1) was not fulfilled. The present data indicate that the experimental facts (1) and (2) are fulfilled in $n$-BuLi/THF, cumyl K/THF, and cumyl Cs/THF systems for PPA, PBA, and PPTA, except for cumyl Cs/ THF/PPA system.

In the case of the exceptional combination of cumyl Cs/THF/PPA system, the polymerization solution maintained a red color for 2 days. If the rate of initiation is much faster than that of propagation, the polymerization rate can be expressed by

$$
-\mathrm{d}[\mathrm{M}] / \mathrm{d} t=k_{\mathrm{ap}}[\mathrm{LE}][\mathrm{M}]
$$

where $k_{\text {ap }}$ is the apparent rate constant; [M] and [LE] are molar concentrations of the monomer and living end, respectively. If eq 1 is integrated, we have

$$
-\ln (1-x)=k_{\mathrm{ap}}[\mathrm{LE}] t
$$

where $x$ is the polymer conversion. $k_{\text {ap }}$ can be determined from eq 2 . We did not specially study the rate of polymerization using a dilatometer. ${ }^{9,19}$ However, we obtained some data for cumyl Cs/THF (Table I) and cumyl $\mathrm{K} /$ THF ( $x=0.28$ and 0.60 for the results of preliminary polymerizations) for PPA. These data are shown in Figure 6. Equation 2 was found to hold at the lower polymer conversion side. The apparent rate constants of the polymerization, $k_{\mathrm{ap}}{ }^{\mathrm{Cs}}$ for the Cs ion and $k_{\mathrm{ap}}{ }^{\mathrm{K}}$ for the $\mathrm{K}$ ion, were determined from the slopes. The results are shown in Table IV.

From a comparison of $k_{\mathrm{ap}}{ }^{\mathrm{K}}$ and $k_{\mathrm{ap}}{ }^{\mathrm{Cs}}$ for PPA with the apparent rate constant of $\mathrm{Na}$ ion for styrene, ${ }^{20} k_{\text {ap }}{ }^{\mathrm{K}}$ and $k_{\text {ap }}{ }^{\mathrm{Cs}}$ were found to be very small. Moreover, $k_{\mathrm{ap}}{ }^{\mathrm{Cs}}$ was about 10 times smaller than $k_{\mathrm{ap}} \mathrm{K}$. This is consistent with the general rule of living anionic polymerization in polar solvents such as THF. ${ }^{20}$ From these results, it is certain that the carbanion of Cs ion for PPA is not deactivated by impurities present in the polymerization solution during the chain propagation reaction. Due to a small $k_{\mathrm{ap}}{ }^{\mathrm{Cs}}$, the polymer conversion of cumyl Cs/ THF/PPA system was less than $85 \%$.

When $n$-BuLi was used as the initiator, the experimental fact (3) was not fulfilled for PPA, PBA, and PPTA, because the $\bar{M}_{w} / \bar{M}_{n}$ values were more than 1.17. In cases of PBA and PPTA, the GPC chromatograms have symmetrical peaks about the elution volume. $N$ Methylpyrrolidine as an additive allowed sharp- 


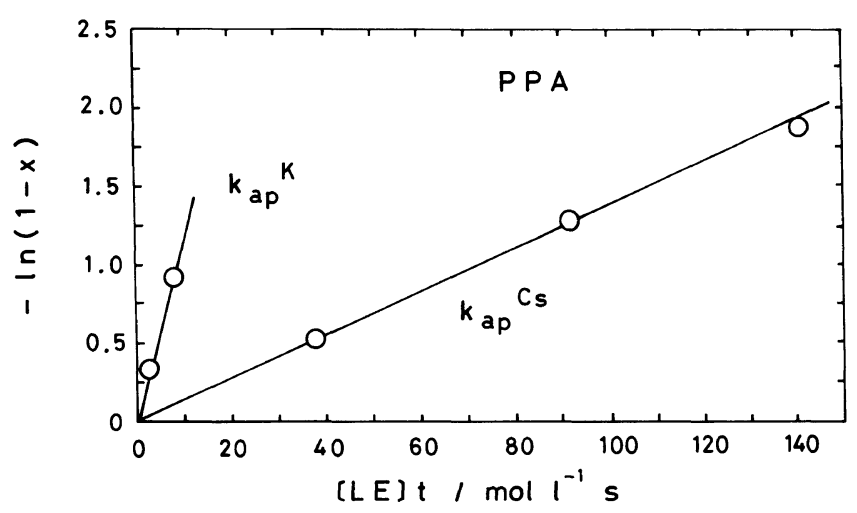

Figure 6. Plots of $-\ln (1-x) v s$. [LE] $t$ for $\operatorname{poly}(N, N$-dimethyl-4-vinylphenylamine), PPA, prepared by using cumyl $\mathrm{K}$ and cumyl $\mathrm{Cs}$ in lower polymer conversion side. The symbols are described in the text. Apparent rate constants of the polymerization are determined from these slopes by use of eq 2 .

Table IV. Apparent rate constants of the living anionic polymerization for PPA The symbols are described in the text

\begin{tabular}{|c|c|c|c|c|}
\hline $10^{2} k_{\text {ap }} \mathrm{Cs}$ & \multirow{2}{*}{$k_{\mathrm{ap}}^{\mathrm{Cs}} / k_{\mathrm{ap}} \mathrm{PSt}^{-\mathrm{Na}^{\mathrm{a}}}$} & \multirow{2}{*}{$\frac{10^{2} k_{\mathrm{ap}}^{\mathrm{K}}}{1 \mathrm{~mol}^{-1} \mathrm{~s}^{-1}}$} & \multirow{2}{*}{ 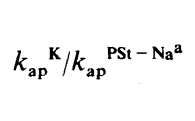 } & \multirow{2}{*}{$k_{\mathrm{ap}}^{\mathrm{K}} / k_{\mathrm{ap}}^{\mathrm{Cs}}$} \\
\hline $1 \mathrm{~mol}^{-1} \mathrm{~s}^{-1}$ & & & & \\
\hline 1.5 & $1.7 \times 10^{-5}$ & 16 & $18 \times 10^{-5}$ & 1.7 \\
\hline
\end{tabular}

ening of the GPC curve of PPTA. The GPC chromatogram of PPA showed tailing at the low moleclar weight side. The additive allowed broadening of the GPC curve. These results suggest that amino groups of the polymers may participate in the solvation of $\mathrm{Li}$ ions during the propagation reaction because of high affinity of amino groups for $\mathrm{Li}$ ion. ${ }^{18}$ Hence, the additive might affect the polymerization mechanism, resulting in change in the shapes of the GPC chromatograms for the polymers prepared. The cumyl Cs/THF system produced a small shoulder at the high molecular weight side for PPT, PBT, and PPTA. The content of the shoulder seemed to increase with increasing concentration of the living ends. Intermolecular reactions of the living ends might occur by termination of Cs ions. However, the shoulder at the high molecular weight side did not remarkably influence the
$\bar{M}_{w} / \bar{M}_{n}$ value, compared with the shoulder at the low molecular weight side.

The cumyl K/THF system did not produce additional shoulders of GPC chromatograms for the three samples. The $\bar{M}_{w} / \bar{M}_{n}$ values for the three samples seemed slightly larger than those for cumyl Cs/THF system. It is, however, difficult to evaluate the difference in the $\bar{M}_{w} / \bar{M}_{n}$ values between $\mathrm{K}$ and Cs ions in this study, because the $\bar{M}_{w} / \bar{M}_{n}$ value is dependent on the molecular weights of the polymers prepared. It was found that the experimental fact (3) was fulfilled for PPA, PBA, and PPTA, when cumyl $\mathrm{K}$ and cumyl $\mathrm{Cs}$ were used as initiators.

We also checked the experimental fact (4) for the combination of cumyl K/THF/PPA system. We succeeded in preparing PPA samples having $\bar{M}_{n}=2.7 \times 10^{4}$ and $\bar{M}_{n}=2.6 \times$ $10^{5}$ : the polymer conversions were $100 \%$, the 
$\bar{M}_{n}$ were in good agreement with the $\bar{M}_{k}$, and the $\bar{M}_{w} / \bar{M}_{n}$ values were less than 1.05 .

\section{REFERENCES}

1. M. Okamura, "Specialty Polymers," N. Ise and I. Tabushi, Ed., Cambridge University Press, New York, N. Y., 1980.

2. K. Se, O. Watanabe, Y. Isono, and T. Fujimoto, Die Makromoleculare Chemie, Macromolecular Symposia Series, 1988, in press.

3. K. Se, K. Miyawaki, and T. Fujimoto, to be published.

4. M. Kijima, K. Se, and T. Fujimoto, to be published.

5. M. Nagasawa and T. Fujimoto, "Progress in Polymer Science Japan," Vol. 3, S. Okamura and M. Takayanagi, Ed., Kodansha Ltd., Tokyo, 1972, p 263.

6. M. Kijima, R. Ohtomo, K. Se, and T. Fujimoto, to be published.

7. K. Yamaguchi, A. Hirao, K. Suzuki, K. Takenaka, S. Nakahama, and N. Yamazaki, J. Polym. Sci., Polym. Lett. Ed., 21, 395 (1983).

8. A. Hirao, K. Takenaka, K. Yamaguchi, N. Yamazaki, and S. Nakahama, Polym. Commun., 24, 339 (1983).

9. Y. Higo, H. Chosi, T. Fujimoto, and M. Nagasawa, Polym. J., 12, 729 (1980).
10. H. Funabashi, Y. Miyamoto, Y. Isono, T. Fujimoto, Y. Matsushita, and M. Nagasawa, Macromolecules, 16, 1 (1983).

11. A. Nakatsuka, T. Kazama, Y. Isono, T. Fujimoto, K. Kato, and M. Suzuki, Kobunshi Ronbunshu, 43 , 627 (1986).

12. F. Hayashi and R. Ichikawa, Kohgyo Kagaku Zasshi, 70, 728 (1969).

13. T. Fujimoto, N. Ozaki, and M. Nagasawa, J. Polym. Sci., A, 3, 2259 (1965).

14. T. Kitano, T. Fujimoto, and M. Nagasawa, Macromolecules, 7, 719 (1974).

15. The Chemical Society of Japan, Ed., "Kagaku Binran: Kisohen," Maruzen, Tokyo, 1984.

16. K. Arai, T. Kotaka, Y. Kitano, and K. Yoshimura, Macromolecules, 13, 1670 (1980).

17. M. Szwarc, "Living Polymers and Mechanisms of Anionic Polymerization," Advanced in Polymer Science, Springer-Verlag, New York, N. Y., 1983.

18. R. N. Young, "Anionic Polymerization of NonPolar Monomers Involving Lithium," Advanced in Polymer Science, Springer-Verlag, New York, N. Y., 1984.

19. T. Kitano, M. Mitsumura, T. Fujimoto, and M. Nagasawa, Macromolecules, 8, 382 (1975).

20. M. Szwarc, "Carbanions Living Polymers and Electron Transfer Processes," Wiley Interscience, New York, N. Y., 1968. 